\title{
New generalized fuzzy metrics and fixed point theorem in fuzzy metric space
}

\section{Robert Plebaniak}

\section{*Correspondence:}

robpleb@math.uni.lodz.pl

Department of Nonlinear Analysis,

Faculty of Mathematics and

Computer Science, University of

Łódź, Banacha 22, Łódź, 90-238,

Poland

\section{空 Springer}

\begin{abstract}
In this paper, in fuzzy metric spaces (in the sense of Kramosil and Michalek (Kibernetika 11:336-344, 1957)) we introduce the concept of a generalized fuzzy metric which is the extension of a fuzzy metric. First, inspired by the ideas of Grabiec (Fuzzy Sets Syst. 125:385-389, 1989), we define a new G-contraction of Banach type with respect to this generalized fuzzy metric, which is a generalization of the contraction of Banach type (introduced by M Grabiec). Next, inspired by the ideas of Gregori and Sapena (Fuzzy Sets Syst. 125:245-252, 2002), we define a new GV-contraction of Banach type with respect to this generalized fuzzy metric, which is a generalization of the contraction of Banach type (introduced by $\vee$ Gregori and A Sapena). Moreover, we provide the condition guaranteeing the existence of a fixed point for these single-valued contractions. Next, we show that the generalized pseudodistance $J: X \times X \rightarrow[0, \infty)$ (introduced by Włodarczyk and Plebaniak (Appl. Math. Lett. 24:325-328, 2011)) may generate some generalized fuzzy metric $N_{\jmath}$ on $X$. The paper includes also the comparison of our results with those existing in the literature.
\end{abstract}

Keywords: fuzzy sets; fuzzy metric space; contraction of Banach type; fixed point; generalized fuzzy metrics; fuzzy metrics

\section{Introduction}

A number of authors generalize Banach's [1] and Caccioppoli's [2] result and introduce the new concepts of contractions of Banach and study the problem concerning the existence of fixed points for such a type of contractions; see e.g. Burton [3], Rakotch [4], Geraghty [5, 6], Matkowski [7-9], Walter [10], Dugundji [11], Tasković [12], Dugundji and Granas [13], Browder [14], Krasnosel'skii et al. [15], Boyd and Wong [16], Mukherjea [17], Meir and Keeler [18], Leader [19], Jachymski [20, 21], Jachymski and Jóźwik [22], and many others not mentioned in this paper.

In 1975, Kramosil and Michalek [23] introduced the concept of fuzzy metric spaces. It is worth noticing that there exist at least five different concepts of a fuzzy metric space (see Artico and Moresco [24], Deng [25], George and Veeramani [26], Erceg [27], Kaleva and Seikkala [28], Kramosil and Michalek [23]).

In 1989, Grabiec [29] proved an analog of the Banach contraction theorem in fuzzy metric spaces (in the sense of Kramosil and Michalek [23]). In his proof, he used a fuzzy version of Cauchy sequence. It is worth noticing that in the literature in order to prove fixed point theorems in fuzzy metric space, authors used two different types of Cauchy sequences. For

@2014 Plebaniak; licensee Springer. This is an Open Access article distributed under the terms of the Creative Commons Attribution License (http://creativecommons.org/licenses/by/2.0), which permits unrestricted use, distribution, and reproduction in any medium, provided the original work is properly cited. 
details see [30]. The existence of fixed points for maps in fuzzy metric spaces was studied by many authors; see e.g. Gregori and Sapena [31], Miheț [32]. Fixed point theory for contractive mappings in fuzzy metric spaces is closely related to the fixed point theory for the same type of mappings in probabilistic metric spaces of Menger type; see Hadžić [33], Sehgal and Bharucha-Reid [34], Schweizer et al. [35], Tardiff [36], Schweizer and Sklar [37], Qiu and Hong [38], Hong and Peng [39], Mohiuddine and Alotaibi [40], Wang et al. [41], Hong [42], Saadati et al. [43], and many others not mentioned in this paper.

In this paper, in fuzzy metric spaces (in the sense of Kramosil and Michalek [23]), we introduce the concept of a generalized fuzzy metric which is the extension of a fuzzy metric. First, inspired by the ideas of Grabiec [29], we define a new G-contraction of Banach type with respect to this generalized fuzzy metric, which is a generalization of a contraction of Banach type (introduced by M Grabiec). Next, inspired by the ideas of Gregori and Sapena [31], we define a new GV-contraction of Banach type with respect to this generalized fuzzy metric, which is a generalization of a contraction of Banach type (introduced by V Gregori and A Sapena). Moreover, we provide the condition guaranteeing the existence of a fixed point for these single-valued contractions. Next, we show that the generalized pseudodistance $J: X \times X \rightarrow[0, \infty)$ (introduced by Włodarczyk and Plebaniak [44]) may generate some generalized fuzzy metric $N_{J}$ on $X$. Moreover, if we put $J=d$, where $d: X \times X \rightarrow[0, \infty)$ is the usual metric, then $N_{J}$ is a fuzzy metric generated by $d$.

\section{On fixed point theory in Kramosil and Michalek's fuzzy metric spaces and George and Veeramani's fuzzy metric spaces}

To begin with, we recall the concept of a fuzzy metric space, which was introduced by Kramosil and Michalek [23] in 1975.

Definition 2.1 [23] The 3-tuple $(X, M, *)$ is a fuzzy metric space if $X$ is an arbitrary set, $*$ is a continuous t-norm, and $M$ is a fuzzy set in $X^{2} \times[0, \infty)$ satisfying the following conditions:

(M1) $\forall_{x, y \in X}\{M(x, y, 0)=0\}$;

(M2) $\forall_{x, y \in X}\left\{\forall_{t>0}\{M(x, y, t)=1\} \Leftrightarrow x=y\right\}$;

(M3) $\forall_{x, y \in X} \forall_{t>0}\{M(x, y, t)=M(y, x, t)\}$;

(M4) $\forall_{x, y, z \in X} \forall_{t, s>0}\{M(x, z, t+s) \geq M(x, y, t) * M(y, z, s)\}$;

(M5) $M(x, y, \cdot):[0, \infty) \rightarrow[0,1]$ is left-continuous, for all $x, y \in X$.

Then $M$ is called a fuzzy metric on $X$.

Definition 2.2 (I) [29] A sequence $\left(x_{m}: m \in \mathbb{N}\right)$ in $X$ is Cauchy in Grabiec's sense (we say G-Cauchy) if

$$
\forall_{t>0} \forall_{p \in \mathbb{N}}\left\{\lim _{m \rightarrow \infty} M\left(x_{m}, x_{m+p}, t\right)=1\right\} .
$$

(II) [29] A sequence $\left(x_{m}: m \in \mathbb{N}\right)$ in $X$ is convergent to $x \in X$ if

$$
\forall_{t>0}\left\{\lim _{m \rightarrow \infty} M\left(x_{m}, x, t\right)=1\right\},
$$

i.e.,

$$
\forall_{t>0} \forall_{\varepsilon>0} \exists_{m_{0} \in \mathbb{N}} \forall_{m \geq m_{0}}\left\{M\left(x_{m}, x, t\right)>1-\varepsilon\right\} .
$$


Of course, since $*:[0,1] \times[0,1] \rightarrow[0,1]$ is continuous, by (M4) it follows that the limit is uniquely determined.

(III) [29] A fuzzy metric space in which every G-Cauchy sequence is convergent is called complete in Grabiec's sense (G-complete for short).

Some interesting observations on these definitions can be found in [45].

In 1989, Grabiec [29] established the following extension of Banach's result in Kramosil and Michalek's fuzzy metric space.

Theorem 2.1 (Fuzzy Banach contraction theorem, Grabiec [29]) Let $(X, M, *)$ be a Gcomplete fuzzy metric space such that

$$
\forall_{x, y \in X}\left\{\lim _{t \rightarrow \infty} M(x, y, t)=1\right\} .
$$

Let $T: X \rightarrow X$ be a mapping satisfying

(G1) $\exists_{k \in(0,1)} \forall_{x, y \in X} \forall_{t>0}\{M(T(x), T(y), k t) \geq M(x, y, t)\}$.

Then $T$ has a unique fixed point.

Next, we recall the concept of a fuzzy metric space, which was introduced by George and Veeramani [26] in 1994.

Definition 2.3 [26] The 3-tuple $(X, M, *)$ is a fuzzy metric space if $X$ is an arbitrary set, $*$ is a continuous t-norm, and $M$ is a fuzzy set on $X^{2} \times(0, \infty)$ satisfying the following conditions:

(M1) $\forall_{x, y \in X} \forall_{t>0}\{M(x, y, t)>0\}$

(M2) $\forall_{x, y \in X}\left\{\forall_{t>0}\{M(x, y, t)=1\} \Leftrightarrow x=y\right\}$;

(M3) $\forall_{x, y \in X} \forall_{t>0}\{M(x, y, t)=M(y, x, t)\}$;

(M4) $\forall_{x, y, z \in X} \forall_{t, s>0}\{M(x, z, t+s) \geq M(x, y, t) * M(y, z, s)\}$;

(M5) $M(x, y, \cdot):(0, \infty) \rightarrow[0,1]$ is continuous, for all $x, y \in X$.

Then $M$ is called a fuzzy metric on $X$.

Definition 2.4 (I) [26] Let $(X, M, *)$ be a fuzzy metric space. The open ball $B(x, r, t)$ for $t>0$ with center $x \in X$ and radius $r, 0<r<1$, is defined as

$$
B(x, r, t)=\{y \in X: M(x, y, t)>1-r\} .
$$

The family $\{B(x, r, t): x \in X, 0<r<1, t>0\}$ is a neighborhood's system for a Hausdorff topology on $X$, which we call induced by the fuzzy metric $M$.

(II) [31] A sequence $\left(x_{m}: m \in \mathbb{N}\right)$ in $X$ is Cauchy in George and Veeramani's sense (we say GV-Cauchy) if

$$
\forall_{\varepsilon>0} \forall_{t>0} \exists_{m_{0} \in \mathbb{N}} \forall_{n, m \geq m_{0}}\left\{M\left(x_{n}, x_{m}, t\right)>1-\varepsilon\right\} .
$$

(III) [31] A fuzzy metric space in which every GV-Cauchy sequence is convergent is called complete in George and Veeramani's sense (GV-complete for short).

In 2002, Gregori and Sapena [31] established the following extension of Banach's result in George and Veeramani's fuzzy metric spaces. 
Theorem 2.2 (Fuzzy Banach contraction theorem, Gregori and Sapena [31]) Let $(X, M, *)$ be a GV-complete fuzzy metric space in which fuzzy contractive sequences, i.e.,

$$
\exists_{k \in[0,1]} \forall_{t>0} \forall_{m \in \mathbb{N}}\left\{\frac{1}{M\left(x_{m+1}, x_{m+2}, t\right)}-1 \leq k\left(\frac{1}{M\left(x_{m}, x_{m+1}, t\right)}-1\right)\right\},
$$

are GV-Cauchy. Let $T: X \rightarrow X$ be a mapping satisfying

(G2) $\exists_{k \in(0,1)} \forall_{x, y \in X} \forall_{t>0}\left\{\frac{1}{M(T(x), T(y), t)}-1 \leq k\left(\frac{1}{M(x, y, t)}-1\right)\right\}$.

Then $T$ has a unique fixed point.

\section{On generalized fuzzy metric and fixed point theory in Kramosil and Michalek's fuzzy metric spaces and George and Veeramani's fuzzy metric spaces}

Now in Kramosil and Michalek's fuzzy metric space we introduce the concept of a generalized fuzzy metric on $X$. Next, we define a new kind of completeness of the space.

Definition 3.1 Let $(X, M, *)$ be a fuzzy metric space. The map $N$ is said to be a G-generalized fuzzy metric on $X$ if the following three conditions hold:

(N1) $\forall_{x, y, z \in X} \forall_{t, s>0}\{N(x, z, t+s) \geq N(x, y, t) * N(y, z, s)\}$;

(N2) $N(x, y, \cdot):[0, \infty) \rightarrow[0,1]$ is left-continuous, for all $x, y \in X$;

(N3) for any sequences $\left(x_{m}: m \in \mathbb{N}\right)$ and $\left(y_{m}: m \in \mathbb{N}\right)$ in $X$ such that

$$
\forall_{t>0} \forall_{p \in \mathbb{N}}\left\{\lim _{m \rightarrow \infty} N\left(x_{m}, x_{m+p}, t\right)=1\right\}
$$

and

$$
\forall_{t>0}\left\{\lim _{m \rightarrow \infty} N\left(x_{m}, y_{m}, t\right)=1\right\},
$$

we have

$$
\forall_{t>0}\left\{\lim _{m \rightarrow \infty} M\left(x_{m}, y_{m}, t\right)=1\right\} .
$$

Remark 3.1 If $(X, M, *)$ is a fuzzy metric space, then the fuzzy metric $M$ is a G-generalized fuzzy metric on $X$. However, there exists a G-generalized fuzzy metric on $X$ which is not a fuzzy metric on $X$ (for details see Example 4.3).

Definition 3.2 (I) A sequence $\left(x_{m}: m \in \mathbb{N}\right)$ in $X$ is $N$-Cauchy in Grabiec's sense (we say $N$-G-Cauchy) if

$$
\forall_{t>0} \forall_{p \in \mathbb{N}}\left\{\lim _{m \rightarrow \infty} N\left(x_{m}, x_{m+p}, t\right)=1\right\} .
$$

(II) A sequence $\left(x_{m}: m \in \mathbb{N}\right)$ in $X$ is $N$-convergent to $x \in X$ if

$$
\forall_{t>0}\left\{\lim _{m \rightarrow \infty} N\left(x_{m}, x, t\right)=1\right\} .
$$

(III) A fuzzy metric space is called $N$-G-complete if each $N$-G-Cauchy sequence $\left(x_{m}\right.$ : $m \in \mathbb{N})$ in $X$ is $N$-convergent to some $x \in X$ and

(NC) $\forall_{t>0}\left\{\lim _{m \rightarrow \infty} N\left(x_{m}, x, t\right)=\lim _{m \rightarrow \infty} N\left(x, x_{m}, t\right)=1\right\}$. 
Now we prove the auxiliary lemma.

Lemma 3.1 Let $(X, M, *)$ be a fuzzy metric space and let the map $N$ be a G-generalized fuzzy metric on $X$. Then for each $x, y \in X$ the following property holds:

$$
\left\{\forall_{t>0}\{N(x, y, t)=1 \wedge N(y, x, t)=1\} \Rightarrow\{x=y\}\right\} .
$$

Proof Let $x, y \in X$ such that

$$
\forall_{t>0}\{N(x, y, t)=1 \wedge N(y, x, t)=1\}
$$

be arbitrary and fixed. By (N1) and (3.4), we get

$$
\forall_{t>0}\left\{N(x, x, t) \geq N\left(x, y, \frac{t}{2}\right) * N\left(y, x, \frac{t}{2}\right)=1 * 1=1\right\} .
$$

Defining the sequences $\left(x_{m}=x: m \in \mathbb{N}\right)$ and $\left(y_{m}=y: m \in \mathbb{N}\right)$, from (3.5) and (3.4) we have

$$
\forall_{t>0} \forall_{p \in \mathbb{N}}\left\{\lim _{m \rightarrow \infty} N\left(x_{m}, x_{m+p}, t\right)=1\right\}
$$

and

$$
\forall_{t>0}\left\{\lim _{m \rightarrow \infty} N\left(x_{m}, y_{m}, t\right)=1\right\} .
$$

Hence, the properties (3.1) and (3.2) hold. Therefore, by (N3), we see that

$$
\forall_{t>0}\left\{\lim _{m \rightarrow \infty} M\left(x_{m}, y_{m}, t\right)=1\right\}
$$

which, by the definition of the sequences $\left(x_{m}=x: m \in \mathbb{N}\right)$ and $\left(y_{m}=y: m \in \mathbb{N}\right)$, gives

$$
\forall_{t>0}\{M(x, y, t)=1\} .
$$

Hence, by (M2), we conclude that $x=y$.

The main result of the paper is the following.

Theorem 3.1 Let $(X, M, *)$ be a fuzzy metric space, and let $N$ be a G-generalized fuzzy metric on $X$ such that

$$
\forall x, y \in X\left\{\lim _{t \rightarrow \infty} N(x, y, t)=1\right\} .
$$

Let $T: X \rightarrow X$ be an N-G-contraction of Banach type, i.e., $T$ is a mapping satisfying

(B1) $\exists_{k \in(0,1)} \forall_{x, y \in X} \forall_{t>0}\{N(T(x), T(y), k t) \geq N(x, y, t)\}$.

We assume that a fuzzy metric space $(X, M, *)$ is $N$-G-complete. Then $T$ has a unique fixed point $w \in X$, and for each $x \in X$, the sequence $\left(x_{m}=T^{m}\left(x_{0}\right): x_{0}=x, m \in \mathbb{N}\right)$ is convergent to $w$. Moreover, $N(w, w, t)=1$, for all $t>0$. 
Proof The proof will be divided into four steps.

Step I. We see that for each $x \in X$ the sequence $\left(x_{m}=T^{m}\left(x_{0}\right): x_{0}=x, m \in \mathbb{N}\right)$ satisfies

$$
\exists_{k \in(0,1)} \forall_{t>0} \forall_{m \in \mathbb{N}}\left\{N\left(x_{m}, x_{m+1}, k t\right) \geq N\left(x_{0}, x_{1}, \frac{t}{k^{m-1}}\right)\right\} .
$$

Indeed, let $x_{0}=x \in X$ be arbitrary and fixed and let $\left(x_{m}=T^{m}\left(x_{0}\right): m \in \mathbb{N}\right)$. Let $k \in(0,1)$ be as in (B1), and let $m \in \mathbb{N}$ and $t>0$ be arbitrary and fixed. From (B1) we obtain

$$
\begin{aligned}
N\left(x_{m}, x_{m+1}, k t\right) & =N\left(T\left(x_{m-1}\right), T\left(x_{m}\right), k t\right) \geq N\left(x_{m-1}, x_{m}, t\right) \\
& =N\left(T\left(x_{m-2}\right), T\left(x_{m-1}\right), k \frac{t}{k}\right) \geq N\left(x_{m-2}, x_{m-1}, \frac{t}{k}\right) \\
& =N\left(T\left(x_{m-3}\right), T\left(x_{m-2}\right), k \frac{t}{k^{2}}\right) \\
& \geq N\left(x_{m-3}, x_{m-2}, \frac{t}{k^{2}}\right) \\
& \geq \cdots \geq N\left(x_{0}, x_{1}, \frac{t}{k^{m-1}}\right) .
\end{aligned}
$$

Consequently, the property (3.7) holds.

Step II. We see that for each $x \in X$ the sequence $\left(x_{m}=T^{m}\left(x_{0}\right): x_{0}=x, m \in \mathbb{N}\right)$ is N-GCauchy, i.e., it satisfies

$$
\forall_{t>0} \forall_{p \in \mathbb{N}}\left\{\lim _{m \rightarrow \infty} N\left(x_{m}, x_{m+p}, t\right)=1\right\} .
$$

Indeed, let $x_{0}=x \in X$ be arbitrary and fixed and let $\left(x_{m}=T^{m}\left(x_{0}\right): m \in \mathbb{N}\right)$. Let $m, p \in \mathbb{N}$ and $t>0$ be arbitrary and fixed. Then by (N1) and (3.7) we calculate

$$
\begin{aligned}
N\left(x_{m}, x_{m+p}, t\right) & \geq N\left(x_{m}, x_{m+1}, \frac{t}{p}\right) * \stackrel{(p)}{\cdots} * N\left(x_{m+p-1}, x_{m+p}, \frac{t}{p}\right) \\
& \geq N\left(x_{0}, x_{1}, \frac{t}{p k^{m}}\right) * \cdots *(p) * N\left(x_{0}, x_{1}, \frac{t}{p k^{m+p-1}}\right) .
\end{aligned}
$$

Now, using (3.6) we obtain

$$
\lim _{m \rightarrow \infty} N\left(x_{m}, x_{m+p}, t\right) \geq 1 * \stackrel{(p)}{\cdots} * 1=1 .
$$

Thus (3.8) holds.

Step III. Next we see that for each $x \in X$ the sequence $\left(x_{m}=T^{m}\left(x_{0}\right): x_{0}=x, m \in \mathbb{N}\right)$ is convergent to a fixed point of $T$.

Indeed, let $x_{0}=x \in X$ be arbitrary and fixed and let $\left(x_{m}=T^{m}\left(x_{0}\right): m \in \mathbb{N}\right)$. By Step II the sequence $\left(x_{m}: m \in \mathbb{N}\right)$ is $N$-G-Cauchy in $X$. By the $N$-G-completeness of $X$ (Definition 3.2(III)), there exists $w \in X$ such that $\left(x_{m}: m \in \mathbb{N}\right)$ is $N$-convergent to $w$ (i.e., $\left.\forall_{t>0}\left\{\lim _{m \rightarrow \infty} N\left(x_{m}, w, t\right)=1\right\}\right)$. Moreover, by $(\mathrm{NC})$, we get

$$
\forall_{t>0}\left\{\lim _{m \rightarrow \infty} N\left(x_{m}, w, t\right)=\lim _{m \rightarrow \infty} N\left(w, x_{m}, t\right)=1\right\} .
$$


Next, using (N1) and (B1) we calculate

$$
\begin{aligned}
\forall_{t>0} & \forall_{m \in \mathbb{N}}\left\{N(T(w), w, t) \geq N\left(T(w), T\left(x_{m}\right), \frac{t}{2}\right) * N\left(T\left(x_{m}\right), w, \frac{t}{2}\right)\right. \\
& =N\left(T(w), T\left(x_{m}\right), \frac{t}{2}\right) * N\left(x_{m+1}, w, \frac{t}{2}\right) \\
& \left.\geq N\left(w, x_{m}, \frac{t}{2 k}\right) * N\left(x_{m+1}, w, \frac{t}{2}\right)\right\}
\end{aligned}
$$

which, by (3.9), gives

$$
\forall_{t>0}\left\{N(T(w), w, t) \geq \lim _{m \rightarrow \infty} N\left(w, x_{m}, \frac{t}{2 k}\right) * \lim _{m \rightarrow \infty} N\left(x_{m+1}, w, \frac{t}{2}\right)=1 * 1=1\right\} .
$$

Similarly, using (N1) and (B1) we calculate

$$
\begin{aligned}
\forall_{t>0} \forall_{m \in \mathbb{N}}\left\{N(w, T(w), t) \geq N\left(w, T\left(x_{m}\right), \frac{t}{2}\right) * N\left(T\left(x_{m}\right), T(w), \frac{t}{2}\right)\right. \\
\quad=N\left(w, x_{m+1}, \frac{t}{2}\right) * N\left(T\left(x_{m}\right), T(w), \frac{t}{2}\right) \\
\left.\quad \geq N\left(w, x_{m+1}, \frac{t}{2}\right) * N\left(x_{m}, w, \frac{t}{2 k}\right)\right\},
\end{aligned}
$$

which, by (3.9), gives

$$
\forall_{t>0}\left\{N(w, T(w), t) \geq \lim _{m \rightarrow \infty} N\left(w, x_{m+1}, \frac{t}{2}\right) * \lim _{m \rightarrow \infty} N\left(x_{m}, w, \frac{t}{2 k}\right)=1 * 1=1\right\}
$$

Now, from (3.10), (3.11), and Lemma 3.1 we obtain $w=T(w)$, i.e., $w$ is a fixed point of $T$ in $X$. Moreover, by (N1), (3.10), and (3.11), we obtain

$$
\forall_{t>0}\left\{N(w, w, t) \geq N\left(w, T(w), \frac{t}{2}\right) * N\left(T(w), w, \frac{t}{2}\right)=1 * 1=1\right\}
$$

Now, if we define the sequence $\left(y_{m}=w: m \in \mathbb{N}\right)$, then by (3.8) and (3.9) we have

$$
\forall_{t>0} \forall_{p \in \mathbb{N}}\left\{\lim _{m \rightarrow \infty} N\left(x_{m}, x_{m+p}, t\right)=1\right\}
$$

and

$$
\forall_{t>0}\left\{\lim _{m \rightarrow \infty} N\left(x_{m}, y_{m}, t\right)=1\right\}
$$

Therefore (3.1) and (3.2) hold, so by (N3) we have $\forall_{t>0}\left\{\lim _{m \rightarrow \infty} M\left(x_{m}, y_{m}, t\right)=1\right\}$, which gives

$$
\forall t>0\left\{\lim _{m \rightarrow \infty} M\left(x_{m}, w, t\right)=1\right\}
$$

Step IV. Finally we see that $w$ is a unique fixed point of $T$ in $X$ and $N(w, w, t)=1$, for all $t>0$. 
Indeed, assume that $T(v)=v$ for some $v \in X$. Then using (B1) we obtain

$$
\begin{aligned}
& \forall_{t>0} \forall_{m \in \mathbb{N}}\{1 \geq N(v, w, t)=N(T(v), T(w), t) \geq N\left(v, w, \frac{t}{k}\right)=N\left(T(v), T(w), \frac{t}{k}\right) \\
&\left.\geq N\left(v, w, \frac{t}{k^{2}}\right) \geq \cdots \geq N\left(v, w, \frac{t}{k^{m}}\right)\right\},
\end{aligned}
$$

which, by (N2) and (3.6), gives

$$
\forall_{t>0}\left\{1 \geq N(v, w, t) \geq \lim _{m \rightarrow \infty} N\left(v, w, \frac{t}{k^{m}}\right)=1\right\} .
$$

Similarly, using (B1), (N3), and (3.6) we calculate $\forall_{t>0}\left\{1 \geq N(w, v, t) \geq \lim _{m \rightarrow \infty} N(w, v\right.$, $\left.\left.\frac{t}{k^{m}}\right)=1\right\}$. Hence,

$$
\forall_{t>0}\{N(v, w, t)=1 \wedge N(w, v, t)=1\} .
$$

Next, applying Lemma 3.1, we get $v=w$, thus the fixed point of $T$ is unique. Moreover, by (3.12) we get $\forall_{t>0}\{N(w, w, t)=1\}$.

Remark 3.2 It is worth noticing that in George and Veeramani's fuzzy metric space we may introduce the concept of a generalized fuzzy metric (in the sense of GeorgeVeeramani) on $X$ (for short, GV-generalized fuzzy metric). Let $(X, M, *)$ be a fuzzy metric space. The map $N$ is said to be a $G V$-generalized fuzzy metric on $X$ if the following three conditions hold:

$\left(\mathrm{N}_{\mathrm{GV}} 1\right) \quad \forall_{x, y, z \in X} \forall_{t, s>0}\{N(x, z, t+s) \geq N(x, y, t) * N(y, z, s)\}$

$\left(\mathrm{N}_{\mathrm{GV}} 2\right) N(x, y, \cdot):(0, \infty) \rightarrow[0,1]$ is continuous, for all $x, y \in X$;

$\left(\mathrm{N}_{\mathrm{GV}} 3\right)$ for any sequences $\left(x_{m}: m \in \mathbb{N}\right)$ and $\left(y_{m}: m \in \mathbb{N}\right)$ in $X$ such that

$$
\forall_{\varepsilon>0} \forall_{t>0} \exists_{m_{0} \in \mathbb{N}} \forall_{n>m \geq m_{0}}\left\{N\left(x_{n}, x_{m}, t\right)>1-\varepsilon\right\}
$$

and

$$
\forall_{t>0} \forall_{\varepsilon>0} \exists_{m_{0} \in \mathbb{N}} \forall_{m \geq m_{0}}\left\{N\left(x_{m}, y_{m}, t\right)>1-\varepsilon\right\}
$$

we have

$$
\forall_{t>0} \forall_{\varepsilon>0} \exists_{m_{0} \in \mathbb{N}} \forall_{m \geq m_{0}}\left\{M\left(x_{m}, y_{m}, t\right)>1-\varepsilon\right\}
$$

Remark 3.3 Using similar considerations, we may introduce the concepts of $N$-Cauchy sequences in George and Veeramani's sense and N-GV-completeness. Precisely: (I) A sequence $\left(x_{m}: m \in \mathbb{N}\right)$ in $X$ is $N$-Cauchy in George and Veeramani's sense (we say $N$-GVCauchy) if

$$
\forall_{\varepsilon>0} \forall_{t>0} \exists_{m_{0} \in \mathbb{N}} \forall_{n>m \geq m_{0}}\left\{N\left(x_{m}, x_{n}, t\right)>1-\varepsilon\right\} .
$$

(II) A fuzzy metric space is called $N$-GV-complete, if each $N$-GV-Cauchy sequence $\left(x_{m}\right.$ : $m \in \mathbb{N})$ in $X$ is $N$-convergent to some $x \in X$ and $\forall_{t>0}\left\{\lim _{m \rightarrow \infty} N\left(x_{m}, x, t\right)=\lim _{m \rightarrow \infty} N(x\right.$, $\left.\left.x_{m}, t\right)=1\right\}$. 
Now using similar arguments to the corresponding ones appearing in Section 3 and in the paper of Gregori and Sapena [31] we may conclude the following fixed point theorem in George and Veeramani's fuzzy metric space.

Theorem 3.2 Let $(X, M, *)$ be a fuzzy metric space, and let $N$ be a GV-generalized fuzzy metric on $X^{2} \times[0, \infty)$ such that $N$-fuzzy contractive sequences, i.e.,

$$
\exists_{k \in(0,1)} \forall_{t>0} \forall_{m \in \mathbb{N}}\left\{\frac{1}{N\left(x_{m+1}, x_{m+2}, t\right)}-1 \leq k\left(\frac{1}{N\left(x_{m}, x_{m+1}, t\right)}-1\right)\right\},
$$

are N-GV-Cauchy. Let $T: X \rightarrow X$ be an N-GS-contraction of Banach type (in the sense of Gregori and Sapena), i.e., a mapping satisfying

(B2) $\exists_{k \in(0,1)} \forall_{x, y \in X} \forall_{t>0}\left\{\frac{1}{N(T(x), T(y), t)}-1 \leq k\left(\frac{1}{N(x, y, t)}-1\right)\right\}$.

We assume that a fuzzy metric space $(X, M, *)$ is $N$-GV-complete. Then T has a unique fixed point $w \in X$, and for each $x \in X$, the sequence $\left(x_{m}=T^{m}\left(x_{0}\right): x_{0}=x, m \in \mathbb{N}\right)$, is convergent to $w$. Moreover, $N(w, w, t)=1$, for all $t>0$.

\section{Examples illustrating Theorem 3.2 and some comparisons}

Now, we will present some examples illustrating the concepts that have been introduced so far. We will show a fundamental difference between Theorem 2.2 and Theorem 3.2. Examples will show that Theorem 3.2 is the essential generalization of Theorem 2.2. First, we recall an example of the standard fuzzy metric induced by the metric $d$.

Example 4.1 [31, Definition 2.5] Let $X$ be a metric space. Let $*$ be the usual product on $[0,1]$. Then the 3-tuple $\left(X, M_{d}, *\right)$ where

(MD) $M_{d}(x, y, t)=\frac{t}{t+d(x, y)}, x, y \in X$,

is a George and Veeramani fuzzy metric space (standard fuzzy metric space), and $M_{d}$ is fuzzy metric on $X$.

Recently, in 2011, Włodarczyk and Plebaniak introduced the concept of generalized pseudodistances which, in a natural way, are extensions of metrics. For details see [44]. We recall the concept of a generalized pseudodistance.

Definition 4.1 Let $X$ be a metric space with a metric $d: X \times X \rightarrow[0, \infty)$. The map $J: X \times$ $X \rightarrow[0, \infty)$ is said to be a generalized pseudodistance on $X$ if the following two conditions hold:

(J1) $\forall_{x, y, z \in X}\{J(x, z) \leq J(x, y)+J(y, z)\}$;

(J2) for any sequences $\left(x_{m}: m \in \mathbb{N}\right)$ and $\left(y_{m}: m \in \mathbb{N}\right)$ in $X$ such that

$$
\lim _{n \rightarrow \infty} \sup _{m>n} J\left(x_{n}, x_{m}\right)=0
$$

and

$$
\lim _{m \rightarrow \infty} J\left(x_{m}, y_{m}\right)=0,
$$

we have

$$
\lim _{m \rightarrow \infty} d\left(x_{m}, y_{m}\right)=0 .
$$


We recall also the following remark.

Remark 4.1 (A) If $(X, d)$ is a metric space, then the metric $d: X \times X \rightarrow[0, \infty)$ is a generalized pseudodistance on $X$. However, there exists a generalized pseudodistance on $X$ which is not a metric (see Example 4.2).

(B) From (J1) and (J2) it follows that if $x \neq y, x, y \in X$, then

$$
J(x, y)>0 \vee J(y, x)>0 .
$$

Indeed, if $J(x, y)=0$ and $J(y, x)=0$, then $J(x, x)=0$, since, by (J1), we get $J(x, x) \leq J(x, y)+$ $J(y, x)=0+0=0$. Now, defining $x_{m}=x$ and $y_{m}=y$ for $m \in \mathbb{N}$, we conclude that (4.1) and (4.2) hold. Consequently, by (J2), we get (4.3), which implies $d(x, y)=0$. However, since $x \neq y$, we have $d(x, y) \neq 0$. Contradiction.

(C) From (B) it follows that if $x \neq y$, then

$$
\forall_{x, y \in X}\{\{J(x, y)=0 \wedge J(y, x)=0\} \Rightarrow\{x=y\}\} .
$$

Now we introduce and use some particular kind of generalized pseudodistance to construct the generalized fuzzy metrics.

Example 4.2 Let $X$ be a metric space with metric $d: X \times X \rightarrow[0, \infty)$. Let $E \subset X$ be a bounded and closed set, containing at least two different points, be arbitrary and fixed. Let $c, k>0$ be such that $k>c>\delta(E)$, where $\delta(E)=\sup \{d(x, y): x, y \in E\}$ are arbitrary and fixed. Define the map $J: X \times X \rightarrow[0, \infty)$ as follows:

$$
J(x, y)= \begin{cases}d(x, y) & \text { if }\{x, y\} \cap E=\{x, y\} \\ c & \text { if } x \notin E \wedge y \in E ; \\ k & \text { if } x \in E \wedge y \notin E ; \\ c+k & \text { if }\{x, y\} \cap E=\emptyset .\end{cases}
$$

We can show that the map $J$ is a generalized pseudodistance on $X$. Indeed, let $x, y \in X$ be arbitrary and fixed. We consider the following four cases:

Case 1. If $J(x, y)=d(x, y)$, then by (4.4) we obtain $\{x, y\} \in E$, so by the triangle inequality for $d$, we get $d(x, y) \leq d(x, z)+d(z, y)$ (if $z \in E$ ), and $d(x, y)<c<k=J(x, z)$ (if $z \notin E$, since $c>\delta(E))$. In consequence, in both situations

$$
J(x, y)=d(x, y) \leq J(x, z)+J(z, y) .
$$

Case 2. If $J(x, y)=c$, then by (4.4) we obtain $x \notin E$ and $y \in E$, so by (4.4) $J(x, z)=c$ (if $z \in E$ ) and $J(x, z)=c+k$ (if $z \notin E$ ). In consequence, in both situations

$$
J(x, y)=c \leq J(x, z)+J(z, y) .
$$

Case 3. If $J(x, y)=k$, then by (4.4) we obtain $x \in E$ and $y \notin E$, so by (4.4), $J(z, y)=k$ (if $z \in E$ ) and $J(z, y)=c+k$ (if $z \notin E$ ). In consequence, in both situations

$$
J(x, y)=k \leq J(x, z)+J(z, y) .
$$


Case 4. If $J(x, y)=c+k$, then by (4.4) we obtain $x \notin E$ and $y \notin E$, so by (4.4), $J(x, z)=c$, $J(z, y)=k$ (if $z \in E$ ) and $J(x, z)=J(z, y)=c+k$ (if $z \notin E$ ). In consequence, in both situations

$$
J(x, y)=c+k \leq J(x, z)+J(z, y) .
$$

Therefore, $\forall_{x, y, z \in X}\{J(x, y) \leq J(x, z)+J(z, y)\}$, i.e., the condition (J1) holds.

For proving that $(J 2)$ holds we assume that the sequences $\left(u_{m}: m \in \mathbb{N}\right)$ and $\left(v_{m}: m \in \mathbb{N}\right)$ in $X$ satisfy (4.1) and (4.2). Then, in particular, (4.2) yields

$$
\forall_{0<\varepsilon<c} \exists_{m_{0}=m_{0}(\varepsilon) \in \mathbb{N}} \forall_{m \geq m_{0}}\left\{J\left(v_{m}, u_{m}\right)<\varepsilon\right\} .
$$

By (4.5) and (4.4), since $\varepsilon<c$, we conclude that

$$
\forall_{m \geq m_{0}}\left\{E \cap\left\{v_{m}, u_{m}\right\}=\left\{v_{m}, u_{m}\right\}\right\} .
$$

From (4.6), (4.4), and (4.5), we get $\forall_{0<\varepsilon<c} \exists_{m_{0} \in \mathbb{N}} \forall_{m \geq m_{0}}\left\{d\left(v_{m}, u_{m}\right)<\varepsilon\right\}$. Therefore, the sequences $\left(u_{m}: m \in \mathbb{N}\right)$ and $\left(v_{m}: m \in \mathbb{N}\right)$ satisfy (4.3). Consequently, the property (J2) holds.

In the remaining part of the work, the generalized pseudodistance defined by (4.4) will be called a generalized pseudodistance generated by $d$.

Example 4.3 Let $(X, d)$ be a standard metric space. Let $J: X \times X \rightarrow[0, \infty)$ be a generalized pseudodistance on $X$ generated by $d$ (i.e., defined in Example 4.2). Let $*$ be a continuous t-norm given by $a * b=a b$. Then the $N_{J}$ where

$$
N_{J}(x, y, t)=\frac{t}{t+J(x, y)}
$$

$x, y \in X$, is a GV-generalized fuzzy metric on $X$.

Part I. We prove $\left(\mathrm{N}_{\mathrm{GV}} 1\right)$.

Let $x, y, z \in X$ be arbitrary and fixed. By (J1) we get

$$
J(x, z) \leq J(x, y)+J(y, z) .
$$

Assume that there exist $t_{0}>0$ and $s_{0}>0$ such that $N\left(x, y, t_{0}\right) * N\left(y, z, s_{0}\right)>N\left(x, z, t_{0}+s_{0}\right)$. This, by (4.7), gives

$$
\frac{t_{0}}{t_{0}+J(x, y)} \cdot \frac{s_{0}}{s_{0}+J(y, z)}>\frac{t_{0}+s_{0}}{t_{0}+s_{0}+J(x, z)} .
$$

Hence by a simple calculation we obtain a contradiction. In consequence (N1) and ( $\left.\mathrm{N}_{\mathrm{GV}} 1\right)$ hold.

Part II. We prove $\left(\mathrm{N}_{\mathrm{GV}} 2\right)$.

Let $x, y \in X$ be arbitrary and fixed. Then for $l=J(x, y) \in[0, \infty)$ we have

$$
N_{J}(x, y, t)=\frac{t}{t+l}, \quad t \in[0, \infty)
$$

Thus, $N_{J}(x, y, \cdot):(0, \infty) \rightarrow[0,1]$ is continuous, for each $x, y \in X$. In consequence (N2) and $\left(\mathrm{N}_{\mathrm{GV}} 2\right)$ hold. 
Part III. Next we prove $\left(\mathrm{N}_{\mathrm{GV}} 3\right)$.

We assume that the sequences $\left(x_{m}: m \in \mathbb{N}\right)$ and $\left(y_{m}: m \in \mathbb{N}\right)$ in $X$ satisfy (3.13) and (3.14). Then, in particular, (3.14) yields

$$
\forall_{t>0} \forall_{0<\varepsilon<1-\frac{t}{t+c}} \exists_{m_{0}(\varepsilon) \in \mathbb{N}} \forall_{m \geq m_{0}}\left\{N_{J}\left(x_{m}, y_{m}, t\right)>1-\varepsilon\right\} .
$$

Since $\varepsilon<1-\frac{t}{t+c}$, by a simple calculation we have

$$
1-\varepsilon>\frac{t}{t+c} .
$$

Next, from (4.9) and (4.10) we obtain

$$
\forall_{t>0} \forall_{0<\varepsilon<1-\frac{t}{t+c}} \exists_{m_{0}(\varepsilon) \in \mathbb{N}} \forall_{m \geq m_{0}}\left\{N_{J}\left(x_{m}, y_{m}, t\right)>\frac{t}{t+c}\right\} .
$$

Now, let $m \geq m_{0}$. We obtain $N_{J}\left(x_{m}, y_{m}, t\right)>\frac{t}{t+c}$, next, by (4.7) we have $\frac{t}{t+J\left(x_{m}, y_{m}\right)}>\frac{t}{t+c}$, so $t+J\left(x_{m}, y_{m}\right)<t+c$ and finally $J\left(x_{m}, y_{m}\right)<c$, which, by (4.4), gives $J\left(x_{m}, y_{m}\right)=d\left(x_{m}, y_{m}\right)$. Therefore $N_{J}\left(x_{m}, y_{m}, t\right)=M\left(x_{m}, y_{m}, t\right)$.

Hence, using (4.9) we obtain

$$
\forall_{t>0} \forall_{0<\varepsilon<1-\frac{t}{t+c}} \exists_{m_{0}(\varepsilon) \in \mathbb{N}} \forall_{m \geq m_{0}}\left\{M_{d}\left(x_{m}, y_{m}, t\right)>1-\varepsilon\right\} .
$$

Consequently (3.15) holds. Hence ( $\left.\mathrm{N}_{\mathrm{GV}} 3\right)$ holds.

Example 4.4 Let $\left(X, M_{d}, *\right)$ be a standard fuzzy metric space, where $X=[0,1], *$ be a continuous t-norm given by $a * b=a b$. Let the closed set $E=\left[0, \frac{3}{8}\right] \subset X$ and let $J: X \times X \rightarrow$ $[0, \infty)$ be given by

$$
J(x, y)= \begin{cases}d(x, y) & \text { if }\{x, y\} \cap E=\{x, y\} \\ 2 & \text { if } x \notin E \wedge y \in E ; \\ 3 & \text { if } x \in E \wedge y \notin E ; \\ 5 & \text { if }\{x, y\} \cap E=\varnothing .\end{cases}
$$

Let $N_{J}$ be defined by

$$
N_{J}(x, y, t)=\frac{t}{t+J(x, y)} \text {. }
$$

Let $T: X \rightarrow X$ be a single-valued map given by

$$
T(x)= \begin{cases}0 & \text { for } x \in\left[0, \frac{1}{2}\right] ; \\ \frac{3}{2} x-\frac{3}{4} & \text { for } x \in\left(\frac{1}{2}, \frac{3}{4}\right] ; \quad x \in X \\ \frac{3}{8} & \text { for } x \in\left(\frac{3}{4}, 1\right],\end{cases}
$$

(A) By Example 4.2, $J$ is a generalized pseudodistance on $X$. Next, by Example $4.3, N_{J}$ is a GV-generalized fuzzy metric on $X$.

(B) We observe that $T$ is $N_{J}$-GS-contraction of Banach type, i.e., $T$ satisfies the condition (B2). The proof will be divided into two steps. 
Step I. First, we show that $T$ satisfies the following conditions:

$$
\exists_{\lambda \in[0,1)} \forall_{x, y \in X}\{J(T(x), T(y)) \leq \lambda J(x, y)\} .
$$

Indeed, let $\lambda=\frac{1}{4}$ and let $x, y \in X$ be arbitrary and fixed. We consider the following two cases:

Case 1. If $\{x, y\} \cap E=\{x, y\}$ then by (4.12), $J(x, y)=d(x, y)$. Moreover, since $\max \{x, y\}<\frac{1}{2}$ thus by (4.14), $T(x)=T(y)=0 \in E$. Hence, by (4.12), we obtain

$$
J(T(x), T(y))=J(0,0)=d(0,0)=0 \leq \frac{1}{4} d(x, y)=\lambda J(x, y) .
$$

Case 2. If $\{x, y\} \cap E \neq\{x, y\}$ then by (4.12), $J(x, y) \in\{2,3,5\}$. Moreover, since

$$
\forall_{x, y \in X}\left\{\max \{T(x), T(y)\} \leq \frac{3}{8}\right\}, \quad\{T(x), T(y)\} \cap E=\{T(x), T(y)\},
$$

and by (4.12), $J(T(x), T(y))=d(T(x), T(y))<\delta(E)=3 / 8$. Hence we obtain

$$
J(T(x), T(y))=d(T(x), T(y)) \leq \frac{3}{8}<\frac{1}{2}=\frac{1}{4} \cdot 2=\frac{1}{4} \cdot \min \{2,3,5\} \leq \lambda J(x, y) .
$$

Concluding, from (4.16) and (4.17), we obtain (4.15).

Step II. We show that $T$ satisfies the following conditions:

$$
\exists_{k \in(0,1)} \forall_{x, y \in X} \forall_{t>0}\left\{\frac{1}{N(T(x), T(y), t)}-1 \leq k\left(\frac{1}{N(x, y, t)}-1\right)\right\} .
$$

Let $k=\lambda=\frac{1}{4}$. Let $x, y \in X, t>0$ be arbitrary and fixed. By (4.15) we know that $J(T(x)$, $T(y)) \leq \lambda J(x, y)$. Hence, we obtain the following chain of equivalences:

$$
\begin{aligned}
& \{J(T(x), T(y)) \leq \lambda J(x, y)\} \\
& \quad \Leftrightarrow \quad\left\{\frac{J(T(x), T(y))}{t} \leq k \frac{J(x, y)}{t}\right\} \\
& \quad \Leftrightarrow \quad\left\{\frac{t+J(T(x), T(y))}{t}-1 \leq k\left[\frac{t+J(x, y)}{t}-1\right]\right\} \\
& \quad \Leftrightarrow \quad\left\{\frac{1}{N_{J}(T(x), T(y), t)}-1 \leq k\left[\frac{1}{N_{J}(x, y, t)}-1\right]\right\} .
\end{aligned}
$$

Hence, the condition (4.18) is true, and the map $T$ is $N_{J}$-GS-contraction of Banach type. (C) Observe that $T$ is not contraction of Banach type (in the sense of Gregori and Sapena), i.e., $T$ does not satisfy the condition (G2). Indeed, suppose that $T$ is contraction of Banach type (in the sense of Gregori and Sapena). Then there exists $k_{0} \in[0,1)$ such that

$$
\forall_{t>0} \forall_{x, y \in X}\left\{\frac{1}{M_{d}(T(x), T(y), t)}-1 \leq k_{0}\left[\frac{1}{M_{d}(x, y, t)}-1\right]\right\} .
$$

In particular, for $x_{0}=1 / 2$ and $y_{0}=3 / 4$, by (4.14), we have $T\left(x_{0}\right)=0, T\left(y_{0}\right)=3 / 8$. Hence $d\left(T\left(x_{0}\right), T\left(y_{0}\right)\right)=3 / 8$. Moreover, $d\left(x_{0}, y_{0}\right)=1 / 4$ and consequently, for each $t>0$, by (MD) 
and (4.19), we have

$$
\begin{aligned}
\frac{3 / 8}{t} & =\frac{t+3 / 8}{t}-1=\frac{1}{M_{d}(T(x), T(y), t)}-1 \\
& \leq k_{0}\left[\frac{1}{M_{d}(x, y, t)}-1\right] \\
& =k_{0} \frac{t+1 / 4}{t}-1=k_{0} \frac{1 / 4}{t} .
\end{aligned}
$$

Hence $3 / 2 \leq k_{0}$, which is impossible (recall $k_{0} \in[0,1)$ ).

(D) Now we see that $\left(X, M_{d}, *\right)$ is GV-complete standard fuzzy metric space.

Indeed, we see that $(X, d)$ is complete metric space, thus by [31, Result 4.3] we conclude that the standard fuzzy metric space $\left(X, M_{d}, *\right)$ is GV-complete.

(E) Next, we observe that the fuzzy metric space $(X, M, *)$ is $N$-GV-complete.

Indeed, let $\left(x_{m}: m \in \mathbb{N}\right)$ be a sequence such that $\left(x_{m}: m \in \mathbb{N}\right)$ is $N_{J}$-GV-Cauchy, i.e.,

$$
\forall_{\varepsilon>0} \forall_{t>0} \exists_{m_{0} \in \mathbb{N}} \forall_{n>m \geq m_{0}}\left\{N_{J}\left(x_{m}, x_{n}, t\right)>1-\varepsilon\right\} .
$$

Now, by (4.13) and (4.20) we have

$$
\forall_{\varepsilon>0} \forall_{t>0} \exists_{m_{0} \in \mathbb{N}} \forall_{n>m \geq m_{0}}\left\{\frac{t}{t+J\left(x_{m}, x_{n}, t\right)}>1-\varepsilon\right\} .
$$

Hence, in particular, (4.21) yields

$$
\forall_{t>0} \forall_{0<\varepsilon<\min \left\{\frac{2}{2+t}, 1\right\}} \exists_{m_{0} \in \mathbb{N}} \forall_{n>m \geq m_{0}}\left\{\frac{t}{t+J\left(x_{m}, x_{n}, t\right)}>1-\varepsilon\right\} .
$$

Hence by (4.12) we get

$$
\exists_{m_{0} \in \mathbb{N}} \forall_{n>m \geq m_{0}}\left\{\left\{x_{m}, x_{n}\right\} \cap E=\left\{x_{m}, x_{n}\right\}\right\},
$$

which gives $\forall_{m \geq m_{0}}\left\{x_{m} \in E\right\}$. Moreover, by (4.20), after simple calculations we see that the sequence $\left(x_{m}: m \in \mathbb{N}\right)$ is GV-Cauchy. Now from (D) we obtain the result that there exists $x \in X$ such that

$$
\forall_{t>0}\left\{\lim _{m \rightarrow \infty} M_{d}\left(x_{m}, x, t\right)=1\right\} .
$$

Now, from (4.22) and (MD) we know that $\lim _{m \rightarrow \infty} x_{m}=x$. Moreover, since $E$ is a closed set, we obtain $x \in E$. Hence

$$
\exists_{m_{0} \in \mathbb{N}} \forall_{m \geq m_{0}}\left\{\left\{x_{m}, x\right\} \cap E=\left\{x_{m}, x\right\},\right.
$$

which, by (4.12), gives

$$
\exists_{m_{0} \in \mathbb{N}} \forall_{m \geq m_{0}}\left\{J\left(x_{m}, x\right)=J\left(x, x_{m}\right)=d\left(x_{m}, x\right)\right\} .
$$


Finally, by (4.13) and (4.23) we have $N_{J}\left(x_{m}, x, t\right)=\frac{t}{t+J\left(x_{m}, x\right)}=\frac{t}{t+J\left(x, x_{m}\right)}=N_{J}\left(x, x_{m}, t\right)$. Hence, by (4.23) we obtain

$$
\begin{gathered}
\forall_{t>0}\left\{\lim _{m \rightarrow \infty} N_{J}\left(x_{m}, x, t\right)=\lim _{m \rightarrow \infty} N_{J}\left(x, x_{m}, t\right)=\lim _{m \rightarrow \infty} \frac{t}{t+J\left(x, x_{m}\right)}\right. \\
\left.=\lim _{m \rightarrow \infty} \frac{t}{t+d\left(x, x_{m}\right)}=\lim _{m \rightarrow \infty} M_{d}\left(x, x_{m}, t\right)=1\right\} .
\end{gathered}
$$

Hence we find that $(X, M, *)$ is $N$-GV-complete.

(F) Now we see that each $N$-fuzzy contractive sequence $\left(x_{m}: m \in \mathbb{N}\right)$ is $N$-GV-Cauchy. Indeed, let $\left(x_{m}: m \in \mathbb{N}\right)$ be an $N$-fuzzy contractive sequence, i.e.,

$$
\exists_{k \in(0,1)} \forall_{t>0} \forall_{m \in \mathbb{N}}\left\{\frac{1}{N\left(x_{m+1}, x_{m+2}, t\right)}-1 \leq k\left(\frac{1}{N\left(x_{m}, x_{m+1}, t\right)}-1\right)\right\} .
$$

Hence,

$$
\exists_{k \in(0,1)} \forall_{t>0} \forall_{m \in \mathbb{N}}\left\{\frac{t+J\left(x_{m+1}, x_{m+2}\right)}{t}-1 \leq k\left(\frac{t+J\left(x_{m}, x_{m}\right)}{t}-1\right)\right\}
$$

which gives

$$
\exists_{k \in(0,1)} \forall_{t>0} \forall_{m \in \mathbb{N}}\left\{J\left(x_{m+1}, x_{m+2}\right) \leq k J\left(x_{m}, x_{m+1}\right)\right\} .
$$

Now, by (4.12),

$$
\exists_{m_{0} \in \mathbb{N} ; m_{0} \leq 4} \forall_{m \geq m_{0}}\left\{x_{m} \in E\right\}
$$

and

$$
\exists_{k \in(0,1)} \forall_{t>0} \forall_{m \geq m_{0}}\left\{d\left(x_{m+1}, x_{m+2}\right) \leq k d\left(x_{m}, x_{m+1}\right)\right\} .
$$

Hence, the sequence $\left(x_{m}: m \in \mathbb{N}\right)$ is contractive in $(X, d)$, thus (by the completeness of $(X, d))$ convergent. Consequently, $\left(x_{m}: m \in \mathbb{N}\right)$ is Cauchy in $X$. Therefore $\left(x_{m}: m \in \mathbb{N}\right)$ is GV-Cauchy in $\left(X, M_{d}, *\right)$, i.e.,

$$
\forall_{\varepsilon>0} \forall_{t>0} \exists_{m_{1} \in \mathbb{N}} \forall_{n, m \geq m_{1}}\left\{M_{d}\left(x_{n}, x_{m}, t\right)>1-\varepsilon\right\} .
$$

Now let $t>0$ and $\varepsilon>0$ be arbitrary and fixed. Then there exists $m_{2}=\max \left\{m_{0}, m_{1}\right\}$ such that, by (4.24) and (4.25), we obtain

$$
\forall_{n>m \geq m_{2}}\left\{N_{J}\left(x_{n}, x_{m}, t\right)=M_{d}\left(x_{n}, x_{m}, t\right)>1-\varepsilon\right\} .
$$

Hence the sequence $\left(x_{m}: m \in \mathbb{N}\right)$ is $N$-GV-Cauchy.

(G) Finally, we observe that all assumptions of Theorem 3.2 are satisfied. The point $w=0$ is a fixed point of $T$ in $X$. Moreover, for each $x \in X$, the sequence $\left(x_{m}=T^{m}\left(x_{0}\right): x_{0}=x, m \in\right.$ $\mathbb{N})$ satisfies condition $\forall_{m \geq 3}\left\{x_{m}=0\right\}$. Hence, by (MD), we obtain $\forall_{t>0}\left\{\lim _{m \rightarrow \infty} M\left(x_{m}, x, t\right)=\right.$ $\left.\lim _{m \rightarrow \infty} \frac{t}{t+d\left(0, x_{m}\right)}=1\right\}$. In consequence, for each $x \in X$, the sequence $\left(x_{m}=T^{m}\left(x_{0}\right): x_{0}=\right.$ $x, m \in \mathbb{N}$ ) is convergent (in the standard fuzzy metric space $\left.\left(X, M_{d}, *\right)\right)$ to $w$. 
Remark 4.2 (I) We observe that if we put $N_{J}=M_{d}$ in Theorem 3.2, then we find that Theorems 3.2 and 2.2 are identical.

(II) The introduction of the concept of a generalized fuzzy metric is essential. If $X$ and $T$ are such as in Example 4.4, then we can show that $T$ is an $N_{J}$-GS-contraction of Banach type, but it is not a contraction of Banach type with respect to $M_{d}$ (see Example 4.4(B), (C)). Hence, we see that our theorem is a generalization of Theorem 2.2 (Gregori and Sapena [31]).

\section{Competing interests}

The author declares that they have no competing interests.

Received: 3 June 2014 Accepted: 20 November 2014 Published: 08 Dec 2014

\section{References}

1. Banach, S: Sur les opérations dans les ensembles abstraits et leurs applications aux équations intégrales. Fundam. Math. 3, 133-181 (1922)

2. Caccioppoli, R: Un teorema generale sull'esistenza di elementi uniti in una trasformazione funzionale. Atti Accad. Naz. Lincei, Rend. Cl. Sci. Fis. Mat. Nat. 11, 794-799 (1930)

3. Burton, TA: Integral equations, implicit functions, and fixed points. Proc. Am. Math. Soc. 124, $2383-2390$ (1996)

4. Rakotch, E: A note on contractive mappings. Proc. Am. Math. Soc. 13, 459-465 (1962)

5. Geraghty, MA: An improved criterion for fixed points of contractions mappings. J. Math. Anal. Appl. 48, 811-817 (1974)

6. Geraghty, MA: On contractive mappings. Proc. Am. Math. Soc. 40, 604-608 (1973)

7. Matkowski, J: Integrable solution of functional equations. Diss. Math. 127, 1-68 (1975)

8. Matkowski, J: Fixed point theorems for mappings with a contractive iterate at a point. Proc. Am. Math. Soc. 62, 344-348 (1977)

9. Matkowski, J: Nonlinear contractions in metrically convex space. Publ. Math. (Debr.) 45, 103-114 (1994)

10. Walter, W: Remarks on a paper by F. Browder about contraction. Nonlinear Anal. 5, 21-25 (1981)

11. Dugundji, J: Positive definite functions and coincidences. Fundam. Math. 90, 131-142 (1976)

12. Tasković, MR: A generalization of Banach's contractions principle. Publ. Inst. Math. (Belgr.) 23(37), 171-191 (1978)

13. Dugundji, J, Granas, A: Weakly contractive maps and elementary domain invariance theorems. Bull. Greek Math. Soc. 19, 141-151 (1978)

14. Browder, FE: On the convergence of successive approximations for nonlinear equations. Indag. Math. 30, 27-35 (1968)

15. Krasnosel'skiĭ, MA, Vănikko, GM, Zabrě̌ko, PP, Rutitskiii, YB, Stetsenko, VY: Approximate Solution of Operator Equations. Noordhoof, Groningen (1972)

16. Boyd, DW, Wong, JSW: On nonlinear contractions. Proc. Am. Math. Soc. 20, 458-464 (1969)

17. Mukherjea, A: Contractions and completely continuous mappings. Nonlinear Anal. 1, 235-247 (1977)

18. Meir, A, Keeler, E: A theorem on contraction mappings. J. Math. Anal. Appl. 28, 326-329 (1969)

19. Leader, S: Equivalent Cauchy sequences and contractive fixed points in metric spaces. Stud. Math. 66, 63-67 (1983)

20. Jachymski, J: Equivalence of some contractivity properties over metrical structures. Proc. Am. Math. Soc. 125, 2327-2335 (1997)

21. Jachymski, J: On iterative equivalence of some classes of mappings. Ann. Math. Sil. 13, 149-165 (1999)

22. Jachymski, J, Jóźwik, I: Nonlinear contractive conditions: a comparison and related problems. In: Fixed Point Theory and Its Applications. Banach Center Publ., vol. 77, pp. 123-146. Polish Acad. Sci., Warsaw (2007)

23. Kramosil, I, Michalek, J: Fuzzy metric and statistical metric spaces. Kibernetika 11, 336-344 (1975)

24. Artico, G, Moresco, R: On fuzzy metrizability. J. Math. Anal. Appl. 107, 144-147 (1985)

25. Deng, Z: Fuzzy pseudo-metric spaces. J. Math. Anal. Appl. 86, 74-95 (1982)

26. George, A, Veeramani, P: On some results in fuzzy metric spaces. Fuzzy Sets Syst. 64, 395-399 (1994)

27. Erceg, MA: Metric spaces in fuzzy set theory. J. Math. Anal. Appl. 69, 205-230 (1979)

28. Kaleva, O, Seikkala, S: On fuzzy metric spaces. Fuzzy Sets Syst. 12, 215-229 (1984)

29. Grabiec, M: Fixed points in fuzzy metric spaces. Fuzzy Sets Syst. 125, 385-389 (1989)

30. Vasuki, R, Veeramani, P: Fixed point theorems and Cauchy sequences in fuzzy metric spaces. Fuzzy Sets Syst. 135 , 415-417 (2003)

31. Gregori, V, Sapena, A: On fixed-point theorems in fuzzy metric spaces. Fuzzy Sets Syst. 125, 245-252 (2002)

32. Miheț, D: A Banach contraction theorem in fuzzy metric spaces. Fuzzy Sets Syst. 144, 431-439 (2004)

33. Hadžić, O: Fixed point theory in probabilistic metric spaces. Serbian Academy of Sciences and Arts, Branch in Novi Sad, University of Novi Sad, Institute of Mathematics, Novi Sad (1995)

34. Sehgal, VM, Bharucha-Reid, AT: Fixed points of contraction mappings on PM-spaces. Math. Syst. Theory 6, 97-100 (1972)

35. Schweizer, B, Sherwood, H, Tardiff, RM: Contractions on PM-spaces: examples and counterexamples. Stochastica 12(1), 5-17 (1988)

36. Tardiff, RM: Contraction maps on probabilistic metric spaces. J. Math. Anal. Appl. 165, 517-523 (1992)

37. Schweizer, B, Sklar, A: Statistical metric spaces. Pac. J. Math. 10, 314-334 (1960)

38. Qiu, Z, Hong, SH: Coupled fixed points for multivalued mappings in fuzzy metric spaces. Fixed Point Theory Appl. 2013, 162 (2013) 
39. Hong, SH, Peng, Y: Fixed points of fuzzy contractive set-valued mappings and fuzzy metric completeness. Fixed Point Theory Appl. 2013, 276 (2013)

40. Mohiuddine, SA, Alotaibi, A: Coupled coincidence point theorems for compatible mappings in partially ordered intuitionistic generalized fuzzy metric spaces. Fixed Point Theory Appl. 2013, 265 (2013)

41. Wang, S, Alsulami, SM, Ćirić, L: Common fixed point theorems for nonlinear contractive mappings in fuzzy metric spaces. Fixed Point Theory Appl. 2013, 191 (2013)

42. Hong, S: Fixed points for modified fuzzy $\psi$-contractive set-valued mappings in fuzzy metric spaces. Fixed Point Theory Appl. 2014, 12 (2014)

43. Saadati, R, Kumam, P, Jung, SY: On the tripled fixed point and tripled coincidence point theorems in fuzzy normed spaces. Fixed Point Theory Appl. 2014, 136 (2014)

44. Włodarczyk, K, Plebaniak, R: A fixed point theorem of Subrahmanyam type in uniform spaces with generalized pseudodistances. Appl. Math. Lett. 24, 325-328 (2011). doi:10.1016/j.aml.2010.10.015

45. Tirado, P: On compactness and G-completeness in fuzzy metric spaces. Iran. J. Fuzzy Syst. 9(4), 151-158 (2012)

10.1186/1687-1812-2014-241

Cite this article as: Plebaniak: New generalized fuzzy metrics and fixed point theorem in fuzzy metric space. Fixed Point Theory and Applications 2014, 2014:241

\section{Submit your manuscript to a SpringerOpen ${ }^{\circ}$ journal and benefit from:}

- Convenient online submission

- Rigorous peer review

- Immediate publication on acceptance

- Open access: articles freely available online

- High visibility within the field

- Retaining the copyright to your article 